Utah State University

DigitalCommons@USU

1998

\title{
On the Irrelevance of Collusion in Perfectly Correlated Environments
}

Amitrajeet A. Batabyal

Utah State University

Follow this and additional works at: https://digitalcommons.usu.edu/eri

\section{Recommended Citation}

Batabyal, Amitrajeet A., "On the Irrelevance of Collusion in Perfectly Correlated Environments" (1998).

Economic Research Institute Study Papers. Paper 146.

https://digitalcommons.usu.edu/eri/146

This Article is brought to you for free and open access by the Economics and Finance at DigitalCommons@USU. It has been accepted for inclusion in Economic Research Institute Study Papers by an authorized administrator of DigitalCommons@USU. For more information, please contact digitalcommons@usu.edu.

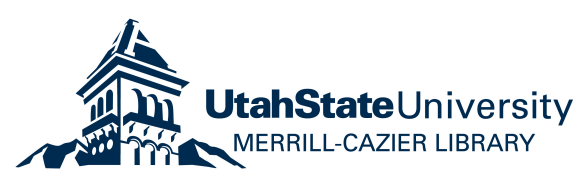


Economic Research Institute Study Paper

ERI \#98-08

\section{ON THE IRRELEVANCE OF COLLUSION IN PERFECTLY CORRELATED ENVIRONMENTS}

by

AMITRAJEET A. BATABYAL

Department of Economics

3530 Old Main Hill

Utah State University

Logan, UT 84322-3530

May 1998 


\title{
ON THE IRRELEVANCE OF COLLUSION IN PERFECTLY CORRELATED ENVIRONMENTS
}

\author{
Amitrajeet A. Batabyal, Associate Professor \\ Department of Economics \\ 3530 Old Main Hill \\ Utah State University \\ Logan, UT 84322-3530
}

The analyses and views reported in this paper are those of the author(s). They are not necessarily endorsed by the Department of Economics or by Utah State University.

Utah State University is committed to the policy that all persons shall have equal access to its programs and employment without regard to race, color, creed, religion, national origin, sex, age, marital status, disability, public assistance status, veteran status, or sexual orientation.

Information on other titles in this series may be obtained from: Department of Economics, 3530 University Boulevard, Utah State University, Logan, Utah 84322-3530.

Copyright $(\subset) 1998$ by Amitrajeet A. Batabyal. All rights reserved. Readers may make verbatim copies of this document for noncommercial purposes by any means, provided that this copyright notice appears on all such copies. 


\title{
ON THE IRRELEVANCE OF COLLUSION IN PERFECTLY \\ CORRELATED ENVIRONMENTS
}

\section{Amitrajeet A. Batabyal}

\begin{abstract}
I study a class of agency problems that are characterized by the existence of an underlying organizational hierarchy. Specifically, I analyze a two-forked, three-tiered hierarchy, and I show that when the private information of the players in the second and in the third tiers of the hierarchy across the two forks of the hierarchy is perfectly correlated, collusion by the players notwithstanding, the principal can always implement the full information optimum in a Bayes-Nash equilibrium. JEL Classification: C72, D82
\end{abstract}

Key words: collusion, contract, hierarchy 


\section{ON THE IRRELEVANCE OF COLLUSION IN PERFECTLY}

\section{CORRELATED ENVIRONMENTS ${ }^{1}$}

\section{Introduction}

The setting of many agency problems in economics involves the analysis of an organizational hierarchy. Consider the problem of designing an international environmental agreement (IEA); this problem has been studied by Barrett (1994), Batabyal (1996, 1997, 1998a), and Bernauer (1995). This mechanism design question cannot be analyzed meaningfully without studying the hierarchical interactions of three players. These three players include a relevant international institution (the principal), national governments (the intermediaries) and polluting firms (the agents) in the various countries. ${ }^{2}$ As a second example, consider the problem of designing effective rural wage compensation schemes, a problem that has been studied by Bardhan (1984), Basu (1992), and Eswaran and Kotwal (1985), among others. Here the relevant design problem once again involves the analysis of a hierarchy; this hierarchy consists of an absentee landlord (the principal), village level supervisors (the intermediaries), and village tenants (the agents). ${ }^{3}$

\footnotetext{
${ }^{1}$ I acknowledge financial support from the Faculty Research Grant program at Utah State University and the Utah Agricultural Experiment Station, Utah State University, Logan, UT 84322-4810, by way of grant UTA 024. Approved as journal paper No. 6085. I thank Jean-Jacques Laffont and Jacques Lawarree for providing me with access to their unpublished work. I alone am responsible for the contents of this paper.

${ }^{2}$ Note that it is not possible to dispense with the government function. In designing an IEA, international institutions interact with national governments directly and with polluting firms only indirectly. As such, any meaningful analysis of the IEA design question must analyze the role of national governments.

${ }^{3}$ I have in mind situations in which it is not possible —or possible only at great cost— to do away with the supervisory function.
} 
In addition to the existence of an explicit organizational hierarchy, these kinds of problems are characterized by two other attributes which deserve some comment. ${ }^{4}$ First, the players occupying the second and the third tiers of the hierarchy typically possess private information about some aspect of the underlying problem. In the first example above, governments and firms in the individual countries can be expected to possess private information about the available pollution abatement technology. In the second example, the village tenants will have private information about their productivity. As a result, if the underlying hierarchy contains more than one fork, i.e., more than one vertical structure, then the question of possible correlation in the private information of the players across the forks is relevant. Second, whenever the principal grants some authority to another player in the hierarchy, that player may choose to exercise the authority for a purpose other than the one intended by the principal. In other words, potential collusion by the players occupying the second and the third tiers of the hierarchy becomes an issue. With regard to my earlier examples, governments and firms within a country may collude to thwart the objectives of an international institution, ${ }^{5}$ or tenants and supervisors within a village may collude to the detriment of the absentee landlord.

Given this background, I propose to extend the hierarchies literature by formally analyzing a two-forked, three-tiered hierarchy with five players. Specifically, I shall show that when the private information of the players in the second and in the third tiers of the hierarchy across the two

${ }^{4}$ For other examples involving the analysis of hierarchies, see Tirole (1994), and Laffont and Martimort(1996).

${ }^{5}$ See Peterson (1993) for a discussion of some practical instances of possible government/firm collusion in an international setting. 
forks of the hierarchy is perfectly correlated, collusion notwithstanding, the principal can always implement the full information optimum in a Bayes-Nash equilibrium.

In other words, in perfectly correlated environments, the principal can engage in relative performance evaluation to effectively extract all the surplus from the players in the second and the third tiers of the two-forked hierarchy. To comprehend the assumption of perfect correlation, recall the two examples discussed above. With regard to the first example, I have in mind polluting firms in countries with very similar pollution abatement technologies (Norway and Sweden). In this situation, the random variables denoting the private information regarding the quality of the two pollution abatement technologies can be thought of as being perfectly correlated. With regard to the second example, I have in mind villages located close to each other. In this setting, the impact of land quality, rainfall, etc., is likely to affect the productivities of crop-growing tenants in any two villages very similarly. Thus, the private information about tenant productivity in the two villages can be expected to be very strongly correlated. Suppose that the main function of the player occupying the second tier of the hierarchy is that of monitoring and then reporting the results of such monitoring to the principal. ${ }^{6}$ Then the private information of these two players can be expected to be strongly correlated as well. Going back to my two examples, intermediaries in similar countries and in similar villages can be expected to have access to monitoring equipment that is of comparable quality. As a result, the random variables denoting the stochastic outcome of monitoring in the two countries or villages will be strongly correlated.

${ }^{6}$ Several papers have adopted this approach. For instance, see Kofman and Lawarree $(1993,1995)$. The reader should note that monitoring/reporting need not be the main function. Later in this section, I shall provide examples in which other tasks are more important. 
In an environment of perfect correlation, multiple equilibria - a major issue in many agency models-pose no problems and hence attention can be restricted to contracts that can be implemented by the principal in a Bayes-Nash equilibrium. As such, issues of augmentation (Mookherjee and Reichelstein 1990) or the design of equivalent dominant strategy mechanisms (Mookherjee and Reichelstein 1992) are not relevant.

Sappington and Demski (1983) have studied ex post ${ }^{7}$ contracting in a two-tiered hierarchy with two states of nature and perfectly correlated private information. They show that the full information optimum can be implemented by the principal in a dominant strategy equilibrium. Three-tiered hierarchies with a single fork have been studied by Tirole (1986), Demski and Sappington (1987), and Kofman and Lawarree (1993). These researchers have studied the properties of the ex post equilibrium contract governing the interaction between a single principal, a single intermediary (often a boss or a manager), and a single agent. Demski and Sappington (1987) and Kofman and Lawarree (1993) assume that monitoring is possible whereas Tirole (1986) assumes that monitoring is impossible.

In this paper, I have three objectives. First, I shall investigate the robustness of the Sappington and Demski (1983) result by analyzing a model with an expanded state space and an increased number of tiers. As indicated in the introductory paragraph to this paper, the principal purpose of this paper is to analyze agency problems that are characterized by the existence of an organizational hierarchy. In these problems, the hierarchical nature of the interaction between the various players is what is of interest. As such, it is not possible to dispense with the middle tier and

\footnotetext{
${ }^{7} \mathrm{By}$ ex post I mean a situation in which some players acquire their private information before contracting.
} 
meaningfully analyze such problems. Two examples of these kinds of problems were provided earlier. Many other examples of such three-tiered hierarchies come to mind. In the context of a manufacturing firm, a commonly existing hierarchy is that of the manager/foreman/worker. In the context of defense procurement, the typical hierarchy is that of the Defense Department/ contractor(s)/subcontractor(s). Finally as a third example, consider the shareholder/manager/worker hierarchy. In all of these examples, the second tier is an essential component of the hierarchy. The manager cannot ignore the foreman, the Defense Department typically does not deal with subcontractors directly, and shareholders do not interact with workers directly.

Due to the relevance of the second tier, and because the players in the second and the third tiers of the hierarchy possess private information, the possibility of collusion by some of these players emerges. National governments and polluting firms within a country may collude against an international institution, the foreman and the worker may collude to the detriment of a manager, and in the context of defense procurement, contractors and subcontractors may collude. Consequently, a significant part of my analysis will consist of analyzing the properties of collusionproof contracts.

Second, I shall study the vertical and horizontal interaction effects that arise from the perfect correlation in the private information of the second- and the third-tier players in this more general model. Third, I shall discuss some conditions under which the first best implementation result of Sappington and Demski (1983) and that of this paper does not hold. The reader will note that in a sense, this paper extends the multiagent contract theory literature vertically and the hierarchies literature laterally. Specifically, I shall study ex post contracting between a single principal, two intermediaries and two agents. The principal's task is to design an incentive compatible, and 
collusion-proof contract, which will extract all the surplus from the intermediaries and the agents in the two-forked, three-tiered hierarchy. I shall focus on the case in which the private information of the intermediaries and the agents in the two forks of the hierarchy is perfectly correlated. In this setting, I shall demonstrate the power of relative performance evaluation.

The rest of this paper is organized as follows. In section 2, I describe the model in detail and then I characterize the first best or full information optimum. In section 3, I analyze the abovedescribed hierarchy with possible collusion by intermediaries and agents in the two forks of the hierarchy. In section 4, I offer some concluding comments.

\section{The Theoretical Framework}

\section{2a. Description of the Model}

Superscripts $A$ and $B$ will denote the two forks and subscripts $i=1, \ldots, 4$ will refer to the state of nature. In what follows, I shall focus on fork $A$. The reader should note that although I am focussing on fork $A$, the analysis is identical for fork $B$. In particular, all the subsequent results for fork $A$ can be obtained for fork $B$ by simply interchanging the two superscripts. Let $\theta^{A}$ denote the uncertainty about agent productivity; $\theta^{A}$ has binary support $\left[\theta^{A L}, \theta^{A H}\right]$, where $\theta^{A H}>\theta^{A L}>0$, and $\Delta \theta^{A} \equiv \theta^{A H}-\theta^{A L}$. I shall refer to $\theta^{A L}$ as the low-productivity parameter and to $\theta^{A H}$ as the highproductivity parameter.

The risk-averse agent in $A$ produces a good whose output and value in state $i$ are denoted by $x_{i}^{A} \geq 0$. The agent chooses action $e_{i}^{A}>0$ in state $i$. The agent's disutility of action is $g\left(e_{i}^{A}\right)$, with $g^{\prime}(\bullet)>0, g^{\prime \prime}(\bullet)>0$, and $g(0)=0$. This agent has a utility function $U\left[T_{i i}^{A}-g\left(e_{i}^{A}\right)\right]$, with $0<\partial U[\bullet] / \partial T_{i i}^{A}<\infty, \forall T_{i i}^{A} . T_{i i}^{A} \geq 0$ is the monetary transfer made by the principal to the $A$ agent 
when he produces $x_{i}^{A}$ and the $B$ agent produces $x_{i}^{B}$. The $A$ agent's reservation utility is $\hat{U}^{A}=U\left[\hat{T}^{A}\right]$; both $\hat{U}^{A}$ and the reservation transfer $\hat{T}^{A}$ are common knowledge.

By employing a monitoring device, the risk-averse intermediary in $A$ receives a signal $s^{A}$ from the agent regarding his private information and then he (the intermediary) provides a report $r^{A}$ to the principal indicating what he observes about the agent's productivity parameter. ${ }^{8}$ In some states of nature, this monitoring device malfunctions and, hence, in these states the intermediary will be unable to provide the principal with a useful report. The intermediary has a strictly concave and differentiable utility function $V\left(G_{i i}{ }^{A}\right)$, where $G_{i i}^{A}$ is the monetary transfer made to the $A$ intermediary when he reports $r_{i}^{A}$ and the $B$ intermediary reports $r_{i}^{B}$. I assume that $0<V^{\prime}\left(G_{i i}^{A}\right)<\infty, \forall G_{i i}^{A}$. The $A$ intermediary's reservation utility is $\hat{V}^{A}=V\left(\hat{G}^{A}\right)$; both $\hat{V}^{A}$ and the reservation transfer $\hat{G}^{A}$ are common knowledge. ${ }^{9}$

The principal is risk neutral and he has a profit function defined over goods that takes the form $\pi=\Sigma_{l}\left(x^{l}-G^{l}-T^{l}\right), l=A, B$. Note that the output produced by the $A$ agent is $x^{A}=e^{A}+\theta^{A}$. As stated, the principal's profit is the difference between the total production of good $x$ and the sum of the intermediary and the agent transfers. The principal designs the main contract, which he offers to the intermediary and the agent. This contract can only be conditioned on what the principal actually observes, i.e., the two intermediary reports $r^{A}$ and $r^{B}$, and the two output levels $x^{A}$ and $x^{B}$.

\footnotetext{
${ }^{8}$ Since my main objective is not to study monitoring, I shall assume that the use of this monitoring device is costless.

${ }^{9}$ The reader will note that the analysis involves both forks. To see this, note that, inter alia, the transfers to the agent and the intermediary have two subscripts. The first subscript corresponds to fork $A$ and the second subscript corresponds to fork $B$.
} 
There are four states of nature, each state occurring with probability $p_{i}>0$, with $\Sigma_{\forall i} p_{i}=1$. The random variables $\theta^{A}$ and $\theta^{B}$ are perfectly correlated. The principal, the intermediary and the agent in $A$ sign the contract holding asymmetric information about $\theta^{A}$. The contract is ex post, i.e., it is signed after the agent has observed $\theta^{A}$. The agent always observes $\theta^{A}$ before choosing his action. The intermediary may or may not observe the agent's private information. This depends on whether the intermediary's monitoring device functions or malfunctions. In other words, the intermediary's signal $s^{A}$ may or may not be informative. I can now characterize the four states. They are:

$\Rightarrow$ State 1: $\theta_{1}^{A}=\theta_{1}^{A L}, \theta_{1}^{B}=\theta_{1}^{B L}, s_{1}^{A}=\theta_{1}^{A L}, s_{1}^{B}=\theta_{1}^{B L}$,

$\Rightarrow$ State 2: $\theta_{2}^{A}=\theta_{2}^{A L}, \theta_{2}^{B}=\theta_{2}^{B L}, s_{2}^{A}=0_{2}^{A}, s_{2}^{B}=0_{2}^{B}$,

$\Rightarrow$ State 3: $\theta_{3}^{A}=\theta_{3}^{A H}, \theta_{3}^{B}=\theta_{3}^{B H}, s_{3}^{A}=0_{3}^{A}, s_{3}^{B}=0_{3}^{B}$,

$\Rightarrow$ State 4: $\theta_{4}^{A}=\theta_{4}^{A H}, \theta_{4}^{B}=\theta_{4}^{B H}, s_{4}^{A}=\theta_{4}^{A H}, s_{4}^{B}=\theta_{4}^{B H}$.

In state 1 , the agent and the intermediary in both forks observe the low productivity parameter and the intermediary monitoring devices function and hence provide useful information. In state 2 , both agents observe the low productivity parameter, but the two intermediaries observe nothing. In this state, both intermediary monitoring devices malfunction. In state 3 , the two agents observe the high productivity parameter, and, once again, the two intermediary monitoring devices malfunction. Finally, in state 4, agents and intermediaries in both forks observe the high productivity parameter. ${ }^{10}$ I shall assume that $p_{1}>p_{2}$ and that $p_{4}>p_{3}$. That is, the two

${ }^{10} \mathrm{I}$ have assumed that the intermediaries always know when their monitoring devices malfunction. More involved formulations in which the intermediaries do not know the states in which their monitoring devices have malfunctioned are possible. These alternate formulations require additional states, and additional constraints on the principal's problem; as such, these formulations make it difficult to obtain concrete results. 
intermediary monitoring devices are reliable in the sense that they are more likely to function than to fail. The reader will note that the imperfect nature of the two monitoring devices results in two additional states, i.e., states 2 and 3.

The timing of the principal/intermediary/agent game is as follows. First, nature reveals $\theta^{A}$ to the agent and the intermediary receives his signal $s^{A}$. Second, the principal offers the contract to the intermediary and to the agent. Third, the agent chooses action $e^{A}$. Fourth, output $x^{A}$ is produced by the agent and the intermediary sends his report $r^{A}$ to the principal, indicating what he observed. Fifth, the principal compensates the intermediary and the agent in $A$ by making transfers $G^{A}\left(x^{A}, x^{B}, r^{A}, r^{B}\right)$ and $T^{A}\left(x^{A}, x^{B}, r^{A}, r^{B}\right)$.

In the remainder of this paper I shall suppose that the principal can verify the veracity of the report $r^{A}$, when $s^{A}=0^{A}$. That is, if $s^{A}$ is noninformative, then the corresponding report $r^{A}$ reflects this fact and the principal can verify that the true facts are indeed as they have been reported. In symbols, $s^{A}=0^{A} \Rightarrow r^{A}=0^{A}$. On the other hand, to keep the principal's problem interesting and to allow for the possibility of intermediary/agent collusion, I shall permit the intermediary to lie and report that his signal is noninformative when in fact such is not the case. ${ }^{11}$ That is, $s^{A}=\theta^{A} \Rightarrow r^{A} \in\left\{\theta^{A}, 0^{A}\right\}$. This completes the description of my model. I now consider the benchmark case in which perfect information is acquired by the principal.

\section{2b. The First Best Optimum}

In this case, the principal observes $\theta_{i}^{A}$ and the agent's action. When this happens, the

\footnotetext{
${ }^{11}$ The reader will note that I have restricted the intermediary's message space in certain states. Specifically, lying by the intermediary is restricted to states 1 and 4 . Put differently, reporting the wrong state of nature is equivalent to obtaining a noninformative signal. A more general model with more states would permit lying in all four states.
} 
principal bypasses the $A$ intermediary and contracts with the $A$ agent directly. Since this intermediary now has no role to play, he receives his reservation transfer $\hat{G}^{A}$, and hence his reservation utility $\hat{V}^{A}$ in all four states. The principal solves

$$
\max _{e^{A}}\left[e^{A}+\theta^{A}-g\left(e^{A}\right)\right] .
$$

The first-order necessary condition requires that

$$
g^{\prime}\left(e_{*}^{A}\right)=1, \forall \theta \text {. }
$$

In other words, in the first best optimum, the marginal disutility of the action is set equal to the marginal profit. The optimal action $e_{*}^{A}$ is the same in all states of nature. The agent receives a transfer which is independent of the state. This transfer equals $\hat{T}^{A}+g_{*}$, where $g_{*}=g\left(e_{*}^{A}\right)$. I can now define the full-information/first-best optimum.

Definition: In the full-information optimum, (i) the intermediary and the agent in each fork are held to their reservation utilities in all states, (ii) equation (2) holds, and (iii) the contract is Pareto efficient in every state.

I now move on to the more interesting case in which the principal cannot determine either the realization of $\theta^{A}$ or the action undertaken by the $A$ agent.

\section{Contracting with Intermediary/Agent Collusion}

Recall that the principal is unable to monitor the activities of intermediaries and agents in $A$ and $B$. Since the principal can never acquire the agent's private information and must rely - in part — on the intermediary's report $r^{A}$ to design the optimal contract, it is of considerable interest to determine the nature of the equilibrium contract that can be implemented by the principal when the 
intermediary and the agent in $A$ collude to maximize the total transfers to be received from the principal.

I shall model collusion between the intermediary and the agent as follows. Before the revelation of $\theta^{A}$ to the agent and at the time of signing the main contract, the agent and the intermediary in each fork sign a secondary contract which entails the offer and acceptance of a monetary bribe from the agent to the intermediary. Naturally, this secondary contract is unobservable by the principal. The bribe can only be conditioned on what the agent and the intermediary both observe, i.e., the bribe is a function of the intermediary's report $r^{4}$ and the agent's output $x^{A}$. With the payment and receipt of the bribe, the agent's total transfer becomes $\left\{T^{A}(\bullet)-b^{A}\left(r^{A}, x^{A}\right)\right\}$ and the intermediary's total transfer becomes $\left\{G^{A}(\bullet)+b^{A}\left(r^{A}, x^{A}\right)\right\}$, where $b^{A}\left(r^{A}, x^{A}\right)$ is the bribe offered by the agent to the intermediary. I shall not be concerned with the question of how the surplus from the bribe is divided. For my purpose, it is only necessary that the bribe be paid by the agent to the intermediary.

Collusion by the intermediary and the agent alters the incentives of the various parties but not - as we shall see — the nature of the optimal contract offered by the principal. To see why the agent in $A$ might want to bribe the intermediary, consider state 4 . In this state, the intermediary is indifferent between reporting that he has observed $\theta^{A H}$ and reporting that he has observed $0^{A}$. The agent, on the other hand, would prefer that the intermediary report $0^{4}$. This is one instance in which a clear rationale exists for the agent to bribe the intermediary.

In order to formulate and solve the principal's problem when there is collusion, I shall follow Tirole $(1986,1988)$. Tirole's method involves imposing constraints in addition to the usual 
participation and incentive compatibility constraints. These additional constraints are designed to preclude intermediary/agent collusion and hence make the main contract collusion-proof. The solution concept that I am using in this paper is that of a Bayes-Nash equilibrium. Consequently, the reader should note that in this section I am considering simultaneous collusion in both forks. The equilibrium contract designed by the principal for $A$ is collusion-proof on the assumption that if the resulting contract were not constrained to be collusion proof, intermediary/agent coalitions would form in both forks. The reader will note that this assumption of "simultaneous collusion" is weaker than the assumption that requires the contract for $A$ to be collusion-proof whether or not there is collusion in $B$.

I can now formulate the principal's problem. The principal solves ${ }^{12}$

$$
\max _{e_{i}^{A}, \bar{G}_{i i}^{A}, \bar{T}_{i i}^{A}} \Sigma_{\forall i} p_{i}\left(e_{i}^{A}+\theta_{i}^{A}-\bar{G}_{i i}^{A}-\bar{T}_{i i}^{A}\right),
$$

subject to (3a) $U\left[\bar{T}_{i i}^{A}-g\left(e_{i}^{A}\right)\right] \geq \hat{U}^{A}, \forall i$, (3b) $V\left(\bar{G}_{i i}^{A}\right) \geq \hat{V}^{A}, \forall i, \quad$ (3c) $p_{2}\left[\bar{T}_{22}^{A}-g\left(e_{2}^{A}\right)\right] \geq p_{2}\left[\bar{T}_{32}^{A}-g\left(e_{3}^{A}+\Delta \theta^{A}\right)\right], \quad(3 \mathrm{~d}) p_{3}\left[\bar{T}_{33}^{A}-g\left(e_{3}^{A}\right)\right] \geq p_{3}\left[\bar{T}_{23}^{A}-g\left(e_{2}^{A}-\Delta \theta^{A}\right)\right]$, $p_{2}\left[\bar{G}_{22}^{A}+\bar{T}_{22}^{A}-g\left(e_{2}^{A}\right)\right] \geq p_{2}\left[G_{32}^{A}+T_{32}^{A}-g\left(e_{3}^{A}+\Delta \theta^{A}\right)\right]$, (3f) $p_{3}\left[\bar{G}_{33}^{A}+\bar{T}_{33}^{A}-g\left(e_{3}^{A}\right)\right] \geq p_{3}\left[\bar{G}_{23}^{A}+\bar{T}_{23}^{A}-g\left(e_{2}^{A}-\Delta \theta^{A}\right)\right]$, (3g) $p_{1}\left[\bar{G}_{11}^{A}+\bar{T}_{11}^{A}-g\left(e_{1}^{A}\right)\right] \geq p_{2}\left[\bar{G}_{22}^{A}+\bar{T}_{22}^{A}-g\left(e_{2}^{A}\right)\right]$, and (3h) $p_{4}\left[\bar{G}_{44}^{A}+\bar{T}_{44}^{A}-g\left(e_{4}^{A}\right)\right] \geq p_{3}\left[\bar{G}_{33}^{A}+\bar{T}_{33}^{A}-g\left(e_{3}^{A}\right)\right]$.

The four constraints in (3a) and in (3b) are the agent and the intermediary ex post participation constraints. These constraints tell us that it must be individually rational for the agent and the intermediary to contract with the principal in every state. Constraints (3c) and (3d) are the agent's incentive compatibility constraints in states 2 and 3, respectively. These constraints arise because the principal has imperfect information about $\theta^{A}$ in these two states. Note that these are

\footnotetext{
${ }^{12}$ The collusion-proof transfers to the intermediary and the agent will be denoted by $\bar{G}_{i i}^{A}$ and $\bar{T}_{i i}^{A}$, respectively.
} 
also the states in which the intermediary's signal is noninformative. Constraint (3c) says that in state 2 , the agent should not claim that the state is 3 . Similarly, (3d) tells us that in state 3 , the agent should not claim that the state is 2 . Constraint (3e) tells us that in state 2, the intermediary should not be able to bribe the agent to take action at the level that is appropriate for state 3. Similarly, (3f) tells us that the intermediary should not be able to bribe the agent to claim that the state is 2 when it is 3 . Constraints ( $3 \mathrm{~g})$ and $(3 \mathrm{~h})$ are the core collusion constraints. Recall that in states 1 and 4 , the intermediary's signal $s^{A}$ is informative. Thus in these two states, the intermediary can hide this fact. Given this, (3g) and (3h) tell us that should the agent bribe the intermediary, then the total sum of the transfers less the disutility of action in states 1 and 4 cannot be less than the corresponding totals in states 2 and 3, respectively. Solving the principal's problem (3) subject to (3a)-(3h), I can state Theorem 1: In the three-tiered hierarchy with intermediary/agent collusion, the principal can implement the full information optimum contract in a Bayes-Nash equilibrium. This contract has the following features: (i) $e_{i}^{A}=e_{*}^{A}, \forall i$, (ii) $\bar{G}_{i i}^{A}=\hat{G}^{A}, \forall i$, (iii) $\bar{T}_{i i}^{A}=\hat{T}^{A}+g_{*}, \forall i$, (iv) only the intermediary and the agent participation constraints bind, and (v) the equilibrium contract is Pareto efficient in all four states.

Proof: See the Appendix.

To intuitively verify that the contract specified in Theorem 1 is individually rational, incentive compatible, and collusion-proof, I shall proceed as follows. I have to show that the constraints (3a)-(3h) are satisfied. First, by part (iv) of the theorem, constraints (3a) and (3b) hold with equality. Hence the contract is individually rational for the intermediary and the agent. Second, because $\bar{T}_{23}^{A}, \bar{T}_{32}^{A}, \bar{G}_{23}^{A}$, and $\bar{G}_{32}^{A}$ are not arguments of the principal's profit function or the 
intermediary and the agent utility functions, they can be set by the principal so as to ensure strict inequality in constraints (3c)-(3f). Hence the contract is incentive compatible for the intermediary and the agent. Finally, by parts (i), (ii), and (iii) of the theorem and the reliability assumptions $p_{1}>p_{2}$ and $p_{4}>p_{3}$, we see that constraints (3g) and (3h) are also satisfied. Hence the contract is collusion-proof.

To check that the contract specified in Theorem 1 does indeed implement the first best, recall the definition of the first-best optimum given in section $2 b$. First, note that the intermediary and the agent are held to their reservation utilities by part (iv) of the Theorem. Second, criterion (ii) of the definition is satisfied because part (i) of the theorem specifies that the first-best action will be taken in every state of nature. Finally, the fact that the contract is Pareto efficient in every state can be deduced from conditions (i), (ii), and (iii) of the Theorem. ${ }^{13}$

If the principal does indeed offer the contract with the characteristics described in Theorem 1, then his total monetary transfers cannot be altered by changing the intermediary's report or the agent's action. In other words, the principal can be sure that his monetary obligations will be those described in Theorem 1. This is because the equilibrium contract is collusion-proof. Alternately put, the principal offers the best contract possible from the set of feasible contracts that are constrained to be collusion-proof.

Theorem 1 says that the principal can implement the full information optimum in a BayesNash equilibrium. This is a strong result and it tells us that the first-best implementation result of Sappington and Demski (1983) generalizes to three-tiered hierarchies. Indeed, we have seen that

\footnotetext{
${ }^{13}$ See the appendix for further details.
} 
when the private information of the intermediaries and the agents in the two forks of the three-tiered hierarchy is perfectly correlated, collusion is irrelevant, and it essentially plays no role in the design of the equilibrium contract since none of the collusion or incentive compatibility constraints bind at the optimum. The "out of equilibrium" payments to the agent and the intermediary satisfy $\bar{T}_{23}^{A}<\left[\hat{T}^{A}+g\left(e_{*}^{A}-\Delta \theta^{A}\right)\right], \bar{T}_{32}^{A}<\left[\hat{T}^{A}+g\left(e_{*}^{A}+\Delta \theta^{A}\right)\right], \bar{G}_{23}^{A}<\left[\hat{G}^{A}+\hat{T}^{A}-\bar{T}_{23}^{A}+g\left(e_{*}^{A}-\Delta \theta^{A}\right)\right]$, and $\bar{G}_{32}^{A}<\left[\hat{G}^{A}+\hat{T}^{A}-\bar{T}_{32}^{A}+g\left(e_{*}^{A}+\Delta \theta^{A}\right)\right]$.

The contract specified in Theorem 1 can be thought of as an incentive scheme, which effectively places the intermediaries and the agents in the two forks in Prisoner's Dilemma games. By carefully selecting the "out of equilibrium" transfers, the principal is able to ensure that misrepresentation of private information does not pay. As such, "telling the truth" is the unique Bayes-Nash equilibrium in the game for the intermediaries and the agents. Further, the equilibrium contract is Pareto efficient in every state, the first-best action is required in every state, and the transfers made to the intermediary and the agent are the same in all four states.

The Prisoner's Dilemma game approach to deterring collusion described in this paper complements the existing literature on collusion in hierarchies. For instance, Kofman and Lawarree (1995 hereafter KL) and Laffont and Martimort (1996, hereafter LM) have both studied hierarchies consisting of a principal, a supervisor (regulator in LM), and an agent (firm in LM). As in this paper, the occupants of the second and the third tiers of the hierarchy, i.e., the supervisor (regulator) and the agent (firm), may collude. KL show that in a number of circumstances, the principal can deter collusion by designing a Prisoner's Dilemma like mechanism. In this mechanism, a second supervisor is brought in, and the principal establishes incentives in such a way that these two supervisors end up policing each other. LM analyze the behavior of two collusive regulators. They 
show that the regulators' access to information introduces increasing returns in the benefit from a side contract (in my case, the bribe) between a regulator and the firm. This fact enables the principal to design a mechanism that is similar to the Prisoner's Dilemma mechanism. In this mechanism, the principal deters collusion by separating the two collusive regulators. As a result, the information available to any one regulator is reduced, the transaction costs of collusion increase, and social welfare rises. Unlike the scenario described in this paper, the KL and the LM papers are not concerned with correlation in the private information of supervisors (regulators) and agents (firms). This is because both of these papers are concerned with a single-forked hierarchy. Further, in the KL paper, both supervisors are identical and they have access to the same information. By explicitly analyzing a second fork, I have shown that the gains from the design of Prisoner's Dilemma type mechanisms can be realized in multiforked environments as well.

The reader should note that recent research ${ }^{14}$ suggests that the first-best implementation result of this paper and that of Sappington and Demski (1983) typically will not hold if the principal's problem is subject to constraints in addition to the participation, incentive compatibility, and collusion constraints. This tells us that the presence of budget balance or "yardstick" constraints can effectively preclude the principal from placing intermediaries and agents in situations like the Prisoner's Dilemma game. In other words, even in perfectly correlated environments, there are limits to the power of relative performance evaluation.

\footnotetext{
${ }^{14}$ See Batabyal (1998b) for further details.
} 


\section{Conclusions}

In this paper I have analyzed a two-forked, three-tiered hierarchy. I focused on the case in which the private information of the players in the second and in the third tiers of the hierarchy is perfectly correlated. I showed that in this setting, the principal loses nothing from his inability to monitor the actions of the intermediaries and the agents. Indeed, the principal can implement the full information/first best optimum in a Bayes-Nash equilibrium even if the intermediaries and the agents collude. 


\section{References}

Bardhan, P.K. 1984. Land, Labor and Rural Poverty. New York: Columbia University Press.

Barrett, S. 1994. Self-Enforcing International Environmental Agreements. Oxford Economic Papers 46:878-94.

Basu, K. 1992. Limited Liability and the Existence of Share Tenancy. Journal of Development Economics 38:203-20.

Batabyal, A.A. 1996. An Agenda for the Design and Study of International Environmental Agreements. Ecological Economics 19:3-9.

Batabyal, A.A. 1997. Developing Countries and Environmental Protection: The Effects of Budget Balance and Pollution Ceiling Constraints. Journal of Development Economics 54:285-305.

Batabyal, A.A. 1998a. Developing Countries and Environmental Protection: The Effects of Ex Ante versus Ex Post Contracting. Journal of Regional Science, forthcoming.

Batabyal, A. A. 1998b. Developing Countries and Environmental Protection: Contract Design in Perfectly Correlated Environments." Mimeo, Utah State University.

Bernauer, T. 1995. The Effect of International Environmental Institutions: How We Might Learn More.” International Organization 49:351-77.

Demski, J., and D. Sappington. 1987. Hierarchical Regulatory Control. RAND Journal of Economics 18:369-83.

Eswaran, M., and A. Kotwal. 1985. A Theory of Two-Tier Labor Markets in Agrarian Economies. American Economic Review 75:162-77.

Kofman, F., and J. Lawarree. 1993. Collusion in Hierarchical Agency. Econometrica 61:629-56.

Kofman, F., and J. Lawarree. 1995 A Prisoner's Dilemma Model of Collusion Deterrence. Journal of Public Economics 59:117-36.

Laffont, J., and D. Martimort. 1996. Separation of Regulators Against Collusive Behavior. Mimeo, IDEI, Toulouse, France.

Mookherjee, D., and S. Reichelstein. 1990. Implementation via Augmented Revelation Mechanisms. Review of Economic Studies 57:453-75. 
Mookherjee, D., and S. Reichelstein. 1992. Dominant Strategy Implementation of Bayesian Incentive Compatible Allocation Rules. Journal of Economic Theory 56:378-99.

Peterson, M. 1993. International Fisheries Management. In P. Haas, R. Keohane, and M. Levy (eds.), Institutions for the Earth, Cambridge, MA: MIT Press.

Sappington, D., and J. Demski. 1983. Multi-Agent Control in Perfectly Correlated Environments. Economics Letters 13:325-30.

Tirole, J. 1986. Hierarchies and Bureaucracies: On the Role of Collusion in Organizations. Journal of Law, Economics, and Organization 2:181-214.

Tirole, J. 1988. The Multicontract Organization. Canadian Journal of Economics 21:459-66.

Tirole, J. 1994. The Internal Organization of the Government. Oxford Economic Papers 46:1-29. 


\section{Appendix $^{15}$}

To verify Theorem 1 , consider the contract specified by the theorem. In this contract, the equilibrium agent and intermediary transfers are $\bar{T}_{i i}^{A}=\hat{T}^{A}+g_{*}, \forall i$, and $\bar{G}_{i i}^{A}=\hat{G}^{A}, \forall i$, respectively. The "out of equilibrium" agent and intermediary transfers satisfy $\bar{T}_{23}^{A}<\left[\hat{T}^{A}+g\left(e_{*}^{A}-\Delta \theta^{A}\right)\right], \bar{T}_{32}^{A}<\left[\hat{T}^{A}+g\left(e_{*}^{A}+\Delta \theta^{A}\right)\right], \bar{G}_{23}^{A}<\left[\hat{G}^{A}+\hat{T}^{A}-\bar{T}_{23}^{A}+g\left(e_{*}^{A}-\Delta \theta^{A}\right)\right]$, and $\bar{G}_{32}^{A}<\left[\hat{G}^{A}+\hat{T}^{A}-\bar{T}_{32}^{A}+g\left(e_{*}^{A}+\Delta \theta^{A}\right)\right]$.

It is straightforward to verify that the optimal Bayes-Nash strategy for the agent and the intermediary in this contract is to always take the first-best action, and to always report the relevant information truthfully. To see why this is so, consider the following line of reasoning.

First, payments of $\bar{T}_{i i}^{A}=\hat{T}^{A}+g_{*}$ to the agent and $\hat{G}^{A}$ to the intermediary ensure that these two players will participate in the contract. Hence, constraints (3a)-(3b) hold with equality and the contract is individually rational for both these players.

Second, because $\bar{T}_{23}^{A}, \bar{T}_{32}^{A}, \bar{G}_{23}^{A}$, and $\bar{G}_{32}^{A}$ do not enter the objective functions of any of the three players, these four "out of equilibrium" transfers can always be chosen by the principal so that all the incentive compatibility constraints - constraints (3c)-(3f) - hold with strict inequality in equilibrium. Thus, the contract is incentive compatible.

Third, under the terms of the contract described in the theorem, the agent always takes the first-best action and the intermediary always reports truthfully. These two features combined with the reliability assumptions, i.e., $p_{1}>p_{2}$ and $p_{4}>p_{3}$, tell us that the collusion request.

\footnotetext{
${ }^{15}$ Details of the Kuhn-Tucker analysis involved in the proof of Theorem 1 are available from the author upon
} 
constraints - constraints (3g)-(3h) - also hold with strict inequality in equilibrium. Thus, the contract is collusion-proof. The reader should note that the result contained in Theorem 1 is not independent of the state probabilities. In particular, this result depends on the state probabilities in the manner specified above. Put differently, for the result in Theorem 1 to hold, we need $p_{1}>p_{2}$ and $p_{4}>p_{3}$.

Fourth, because the equilibrium transfers to the agent are identical, and because the agent always takes the first best action, the marginal rate of substitution between the transfer and the action always equals unity. Consequently, the requirements of the definition in section $2 \mathrm{~b}$ are satisfied. I conclude that the contract specified by Theorem 1 is individually rational, incentive compatible, collusion-proof, and that it implements the first-best/full-information optimum. 